\title{
Analysis of regulatory $T$ cell subsets in the peripheral blood of immunoglobulin A nephropathy (IgAN) patients
}

\author{
S. Yang, B. Chen, J. Shi, F. Chen, J. Zhang and Z. Sun \\ Department of Nephrology, Huaihe Hospital of Henan University, Kaifeng, China \\ Corresponding author: B. Chen \\ E-mail: baopingchencn@126.com
}

Genet. Mol. Res. 14 (4): 14088-14092 (2015)

Received May 14, 2015

Accepted August 6, 2015

Published October 29, 2015

DOI http://dx.doi.org/10.4238/2015.October.29.28

\begin{abstract}
The aim of this study was to investigate the clinical significance of regulatory $T$ cells (Tregs) and its subsets in immunoglobulin A nephropathy (IgAN) patients. Peripheral blood samples of $20 \lg A N$ patients and 20 healthy individuals of similar ages were analyzed. Levels of Tregs and its subsets, namely nTregs and iTregs, were analyzed using flow cytometry. The number of Tregs in IgAN patients was significantly lower than that in the healthy controls. While significant reduction in iTregs primarily contributed to this effect $(P<0.01)$, nTreg levels did not significantly change $(P>0.05)$. The levels of serum IL-17, IL-10 and TGF- $\beta$ were detected by ELISA method. The levels of IL-10 and TGF- $\beta$ in IgAN patients were lower $(P<0.05)$, whereas those of IL-17 in the IgAN group were higher $(P<0.05)$ than those in the controls. In conclusion, the change in Tregs count in the peripheral blood of IgAN patients is mainly caused by the reduction in iTregs, suggesting a substantial role in the prognosis and treatment of IgAN.
\end{abstract}

Key words: Immunoglobulin A nephropathy; Regulatory T cells; Subset 


\section{INTRODUCTION}

Immunoglobulin A nephropathy (IgAN) is the most common form of glomerulonephritis worldwide. The key cause of this disease is the abnormal deposition of IgA in the glomerular mesangial cells. Although its pathogenesis is still unclear, it is believed to be closely linked to immune imbalance in the body. Regulatory $T$ cells (Treg) are involved in inhibiting $T$ cell activation and in developing immune tolerance against autoimmune diseases, tumors, and allografts (Whibley and Gaffen, 2014; Kumar and Subramaniyam, 2015). T helper 17 and Treg cells mutually restrict each other in the body, which helps maintain immune homeostasis (Franzese et al., 2013; Shan et al., 2015). Previous studies have shown that Tregs level was reduced in IgAN patients, which lead to their weakened ability to suppress immune responses. IgAN is known to result in excess immune response to pathogens and increase in cytokine levels (Huang et al., 2010).

Treg can be naturally occurring (nTreg) or induced (iTreg). nTreg are generated in the thymus and play an important role in developing tolerance to autoantigens. nTregs are characterized with $\mathrm{CD}_{45 \mathrm{RA}}{ }^{+-} \mathrm{CD} 25^{\text {hiFOXP }} 3^{\text {hi }} \mathrm{CD} 39^{+} \mathrm{CD} 73^{+1-} \mathrm{CD} 26^{-}$surface markers. Induced regulatory $\mathrm{T}$ cells (iTreg) are produced from IL-10- or TGF- $\beta$-expressing conventional T cells (Tconv) and play a role in immune tolerance to extraneous antigens. Their characteristic surface markers include CD45RA-CD25 ${ }^{10} \mathrm{CD} 122^{+}$FOXP3 ${ }^{10} \mathrm{CD} 39^{+} \mathrm{CD}^{\circ} 3^{+} \mathrm{CD} 26$ - (Hippen et al., 2011; Whiteside et al., 2012; Lin et al., 2013). In order to understand the pathogenic mechanism of Treg in IgAN further, Treg detection, distribution of Treg subsets, and the levels of IL-17, IL-10 and TGF- $\beta$ were analyzed in the peripheral blood of IgAN patients in this study.

\section{SUBJECTS AND METHODS}

\section{Study population}

This study was conducted in accordance with the declaration of Helsinki with the approval of the Ethics Committee of Huaihe Hospital of Henan University. Among 20 participants diagnosed with IgAN in the nephropathy clinic, 12 were male with an average age of 45 and 8 were female with an average age of 48 . Healthy individual as declared by the physical examination center of Huaihe Hospital of Henan University were recruited as controls. Out of 20, 20 were male with an average age of 44 and 8 were female with average age of 48 . Written informed consent was obtained from all participants.

\section{Detection of regulatory $\mathrm{T}$ cells and its subsets}

Peripheral blood sample $(30 \mathrm{~mL})$ was drawn from all the participants after overnight fasting. Of this, $5 \mathrm{~mL}$ was used to detect the serum levels of IL-17, IL-10 and TGF- $\beta$ and $25 \mathrm{~mL}$ was used for heparin (Haipurui Biotechnology Inc., Shenzhen, China) anticoagulation for further quantification of Tregs and Tregs subsets. The serum cytokines IL-17, IL-10 and TGF- $\beta$ were detected using an ELISA Kit (eBioscience, San Diego, CA, USA). Tregs and its subsets were analyzed by flow cytometry (BD Biosciences, New Jersey, USA). Antibodies against CD4, CD25, Foxp3, CD39, CD73 and CD122 were purchased from Santa-Cruz, CA, USA. 


\section{Statistical analysis}

Collected data were processed with SPSS software (SPSS Inc., Chicago, IL, USA). All data are reported as mean \pm standard deviation. $\mathrm{P}<0.05$ was considered statistically significant.

\section{RESULTS}

\section{Quantification of regulatory T cells and its subsets}

The ratios of Treg accounting for total CD4 ${ }^{+} \mathrm{T}$ cells in the peripheral blood were 5.38 and $9.52 \%$ for IgAN patients and control group respectively $(P<0.05)$. Further, the iTreg proportions were calculated as 3.17 and $6.67 \%$ in IgAN and control groups respectively $(P<0.05)$. The difference in $n$ Treg level was not statistically significant between IgAN patients and healthy controls $(P>0.05)$ (Table 1). Our results suggest that the low Treg level in the IgAN group is mainly due to the decrease of iTreg.

Table 1. Treg content in peripheral blood samples of IgAN patients vs that in healthy individuals.

\begin{tabular}{lccc}
\hline Sample group & Treg (\%) & nTreg (\%) & iTreg (\%) \\
\hline IgAN & $10.66 \pm 5.48^{*}$ & $4.35 \pm 0.82$ & $3.57 \pm 0.42^{*}$ \\
Control & $6.42 \pm 1.82$ & $4.13 \pm 0.75$ & $1.67 \pm 0.15$ \\
\hline${ }^{*} P<0.05$. & & &
\end{tabular}

\section{Levels of IL-17, IL-10 and TGF- $\beta$ in peripheral blood}

Results from the ELISA test are presented in Figure 1. The levels of IL-10 and TGF- $\beta$ in the serum of IgAN patients were lower than the control group consistent with Treg levels as expected $(P<0.05)$. However, IL-17 levels were higher in IgAN group. Hence the reduction was not statistically significant $(P>0.05)$.

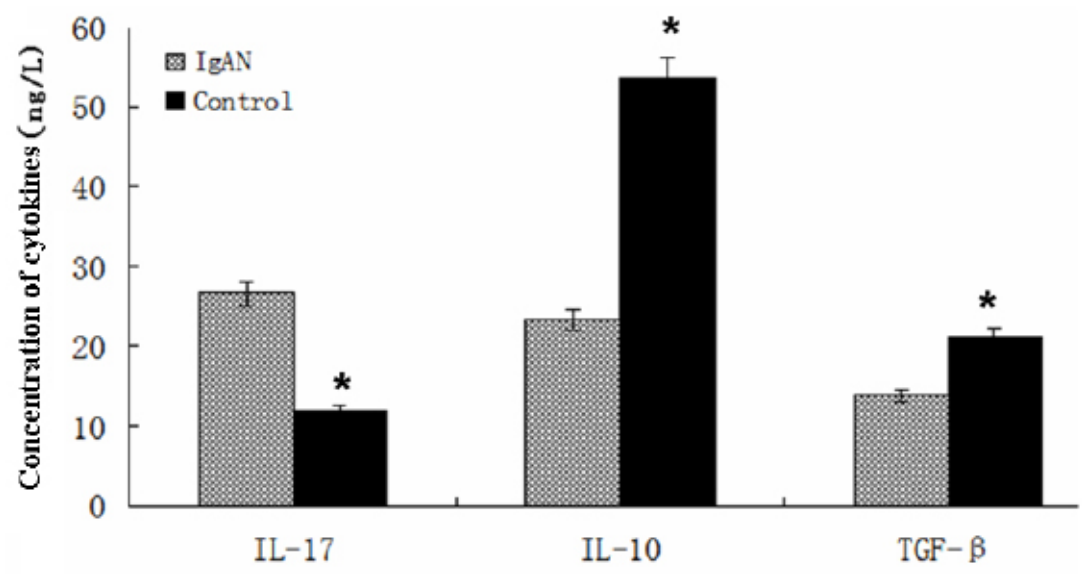

Figure 1. IL-17, IL-10 and TGF- $\beta$ levels in IgAN patients $v s$ healthy individuals $\left({ }^{*} \mathrm{P}<0.05\right)$. 


\section{DISCUSSION}

$\operatorname{lgAN}$, the most common glomerular nephropathy, typically presents itself as macroscopic hematuria associated with proteinuria. In $20-40 \%$ of IgAN patients, this may develop into end-stage renal disease within 25 years after a definite diagnosis. The pathological mechanism underlying $\lg \mathrm{AN}$ is the abnormal glycosylation of IgA1 subtype leading to its polymerization and accumulation in the mesangial cells of the glomerulus upon adsorption. The increase of serum poly $\lg A 1$ (plgA1) and abnormal structure due to low glycosylation are the key pathogenic factors of IgAN (Julian and Novak, 2004). Poly IgA1 is secreted by polyclonal active B cells and the process of $B$ cells secreting IgA is regulated by T cells (Suzuki et al., 2009). Therefore, immune function disorder of $T$ cells may cause the $B$ cells to produce excessive and abnormal IgA, which plays an important role in the pathogenesis of IgAN. CD4+CD25+ regulatory T cells (Treg) are a subset of inhibitory $T$ cells and play an important negative regulatory role in the immune system. It plays significant roles in autoimmunity, immune defense, and immune surveillance through direct or indirect inhibition of proliferation, immunocompetence and intercellular communication of T cells, B cells, dendritic cells (DC), NK cells and monocyte/ macrophage (Wang et al., 2014).

In this study, we compared the number of Treg cells in the peripheral blood of IgAN patients and healthy volunteers of similar age group. The results showed that the number of Tregs in the peripheral blood of IgAN patients was significantly lower than that of healthy volunteers. Tregs are composed of two important subsets namely nTregs and iTregs. Our study showed that the decrease of Tregs in IgAN patients was mainly caused by the decrease of iTregs, whereas $\mathrm{nTregs}$ did not change significantly. A possible explanation is that $\mathrm{nTregs}$ are primarily produced in the thymus and their main physiological function is autoantigen tolerance. Since nTregs can be transformed into iTregs under the effect of some cytokines under certain conditions, distinguishing $\mathrm{nTregs}$ and iTregs could be challenging. However, with the emergence of new markers such as Helios, nTregs or iTregs are easily identified and sorted. Substantial evidence exists to show that sphagitis often leads to the aggravation of IgAN. On one hand, it might be due to the production of IL-17 by Th17 cells. On the other hand, it might lead to the decrease of iTregs, which in turn manifests an immunopathological effect rather than its immune regulatory function (Xie et al., 2004; Meng et al., 2014). The occurrence of Tregs and its subsets in IgAN patients was different from that in tumor patients. Tregs generally exist in the blood and tissue of tumor patients, leading to the inhibition of immune effector cells. Even under the tumor microenvironment, Tconv cells are induced into iTregs further inhibiting anti-tumor immune cells (Whiteside et al., 2012).

Tregs possess considerable plasticity and can be induced into iTregs under the influence of cytokines IL-17, IL-10 or TGF- $\beta$. In this study, the levels of IL-10 and TGF- $\beta$ in the serum of IgAN patients were lower compared to healthy controls. Nevertheless, the serum level of IL-17 in IgAN patients was significantly higher than that of control group. This suggests that Th17 also play an important role in the pathology of IgAN reducing, iTregs level in the peripheral blood of IgAN patients. In conclusion, the low levels of Tregs (especially iTregs), IL-10 and TGF- $\beta$ may influence each other resulting in an imbalance of the immune system in IgAN patients. In addition, these results also suggest that iTregs might have therapeutic effects on IgAN.

\section{Conflicts of interest}

The authors declare no conflict of interest. 


\section{REFERENCES}

Franzese O, Mascali A, Capria A, Castagnola V, et al. (2013). Regulatory T Cells in the immunodiagnosis and outcome of kidney allograft rejection. Clin. Dev. Immunol. 2013: 852395.

Hippen KL, Merke SC, Schirm DK, Nelson C, et al. (2011). Generation and large-scale expansion of human inducible regulatory T cells that suppress graft-versus-host disease. Am. J. Transplant. 11: 1148-1157.

Huang H, Peng Y, Liu H, Yang X, et al. (2010). Decreased CD4+CD25+ cells and increased dimeric IgA-producing cells in tonsils in IgA nephropathy. J. Nephrol. 23: 202-209.

Julian BA and Novak J (2004). IgA nephropathy: an update. Curr. Opin. Nephrol. Hypertens. 13: 171-179.

Kumar P and Subramaniyam G (2015). Molecular underpinnings of Th17 immune-regulation and their implications in autoimmune diabetes. Cytokine 71: 366-376.

Lin XH, Chen MG, Liu Y, Guo ZY, et al. (2013). Advances in distinguishing natural from induced Foxp3+ regulatory T cells. Int. J. Clin. Exp. Pathol. 6: 116-123.

Meng T, Li X, Ao X, Zhong Y, et al. (2014). Hemolytic Streptococcus may exacerbate kidney damage in IgA Nephropathy through CCL20 response to the effect of Th17 Cells. PLoS One 9: e108723.

Shan J, Feng L, Sun G, Chen P, et al. (2015). Interplay between mTOR and STAT5 signaling modulates the balance between regulatory and effective T cells. Immunobiology 220: 510-517.

Suzuki H, Fan R, Zhang Z, Brown R, et al. (2009). Aberrantly glycosylated IgA1 in IgA nephropathy patients is recognized by IgG antibodies with restricted heterogeneity. J. Clin. Invest. 119: 1668-1677.

Wang Y, Wang Y, Wang Y, Zheng G, et al. (2014). Regulatory T cells require renal antigen recognition through the TCR to protect against injury in nephritis. Int. J. Clin. Exp. Pathol. 7: 38-47.

Whibley N and Gaffen SL (2014). Brothers in Arms: Th17 and Treg responses in Candida albicans immunity. PLoS Pathog. 10: e1004456.

Whiteside TL, Schuler P and Schilling B (2012). Induced and natural regulatory T cells in human cancer. Expert Opin. Biol. Ther. 12: 1383-1397.

Xie YS, Chen XM, Nishi S, Narita I, et al. (2004). Relationship between tonsils and IgA nephropathy as well as indications of tonsillectomy. Kidney Int. 65: 1135-1144. 